\title{
The 1-Minute Sit-to-Stand Test in Lung Transplant Candidates: An Alternative to the 6-Minute Walk Test
}

\author{
Dario Kohlbrenner, Christian Benden, and Thomas Radtke
}

\begin{abstract}
BACKGROUND: The 6-min walk test (6MWT) is a well-established functional exercise capacity test in lung transplant candidates. This study aimed to investigate the construct validity of the 1-min sit-to-stand test (1-min STS) as a marker of exercise capacity and knee extensor strength in lung transplantation candidates. METHODS: We retrospectively analyzed data from consecutive subjects referred for lung transplantation evaluation to our institution between 2015 and 2018. RESULTS: 38 subjects were included. We found strong correlations between the normalized 1-min STS and 6MWT $(r=0.79, P<.001)$ and moderate correlations between 1 -min STS and knee extensor strength $(r=0.53, P=.001)$ and between $6 \mathrm{MWT}$ and knee extensor strength $(r=0.44$, $P=.008)$. The 1-min STS elicited greater dyspnea $(P=.008)$ and lower oxygen desaturation compared to the 6MWT $(P<.001)$. CONCLUSIONS: The 1-min STS is a valid functional exercise capacity test in lung transplantation candidates. Due to its ease of application, clinical practitioners may consider using the 1-min STS in situations when the 6MWT cannot be performed. Our pilot study may stimulate future research, including a head-to-head comparison between the 6MWT and 1-min STS in a large patient cohort including post-lung transplantation-monitoring. Key words: lung transplant candidates; functional exercise capacity; construct validity; sit-to-stand test; walk tests; lower limb strength [Respir Care 2020;65(4):437-443. () 2020 Daedalus Enterprises]
\end{abstract}

\section{Introduction}

Evaluation of individuals with end-stage chronic lung disease for lung transplantation is a multimodal and interprofessional procedure, and functional exercise capacity testing is recommended. ${ }^{1}$ Several different tests to assess exercise capacity and select candidates for lung transplantation are available, of which the 6-min walk test (6MWT) is the most commonly applied. ${ }^{1}$ The $6 \mathrm{MWT}$, a submaxi-

\footnotetext{
Mr Kohlbrenner is affiliated with the Division of Pulmonology and Physiotherapy Occupational Therapy, University Hospital Zurich, Switzerland. Dr Benden is affiliated with the Division of Pulmonology, University Hospital Zurich, Switzerland. Dr Radtke is affiliated with the Division of Occupational and Environmental Medicine, Epidemiology, Biostatistics and Prevention Institute, University of Zurich, and University Hospital Zurich, Switzerland.
}

The authors have disclosed no conflicts of interest.

Correspondence: Thomas Radtke PhD, University of Zurich, Hirschengraben 84, 8001 Zurich, Switzerland. E-mail: thomas.radtke@uzh.ch.

DOI: $10.4187 /$ respcare. 07124 mal exercise test, predicts survival in lung transplantation candidates, preferably in combination with other measures, such as the BODE index (body mass index, obstruction, dyspnea, and exercise). ${ }^{1}$ However, the 6MWT requires space (ie, a 30-m hallway) and time, and it is very sensitive to changes in methodology such as supplemental oxygen, track layout and length, and especially variation in verbal instructions. ${ }^{2}$

Growing evidence suggests that the 1-min sit-to-stand test (1-min STS) is an easily applicable and time-saving alternative to assess exercise capacity, and it allows the assessment of individuals in need of segregation due to multidrug-resistant airway pathogens. The 1-min STS is a valid, responsive, and reliable test in individuals with various respiratory diseases, including cystic fibrosis and COPD. ${ }^{3,4}$ In COPD, 1-min STS performance is a strong and independent predictor of 2-y and 5-y mortality. ${ }^{5}$

The 6MWT and 1-min STS measure different aspects of exercise capacity, but both tests yield significant correlations with knee extensor muscle strength. ${ }^{6}$ In individuals with COPD, cystic fibrosis, and interstitial lung disease, moderate to strong correlations between both tests and knee extensor strength have been reported, $, 3,8$ indicating 
that the 1-min STS could be a simple tool to assess functional capacity, particularly in settings where a 6MWT cannot be performed. To date, the 1-min STS has not been compared to the 6MWT in the heterogeneous population of lung transplantation candidates with end-stage pulmonary disease and peripheral muscle weakness.

The primary aim of this study was to investigate the construct validity of the 1-min STS as a marker of functional exercise capacity and knee extensor strength in lung transplantation candidates. We further wanted to compare sensations of dyspnea and leg fatigue, as well as heart rate and oxygen saturation $\left(\mathrm{S}_{\mathrm{pO}_{2}}\right)$ responses between these testing modalities.

\section{Methods}

\section{Study Population}

We retrospectively analyzed data from subjects referred for lung transplantation evaluation to the University Hospital Zurich, in Zurich, Switzerland, between June 2015 and February 2018. All subjects underwent a standardized assessment procedure, including measurement of functional exercise capacity (eg, 6MWT, 1-min STS, and knee extensor strength) following standard clinical practice. Subjects provided written informed consent through a general research agreement. The study was conducted in accordance with the Declaration of Helsinki and approved by the Ethics Committee of the Canton of Zurich (KEK-ZH 2017-00871).

\section{Measurements}

Functional capacity tests were performed on the same day and in the following order: (1) 6MWT, (2) knee extension strength, and (3) 1-min STS. Adequate rest was provided between the tests, allowing $\mathrm{S}_{\mathrm{pO}_{2}}$ and heart rate to return to pre-exercise values. The amount of oxygen supplementation, if required, was kept equal during the 6MWT and 1-min STS, respectively.

The 6MWT was performed according to technical standards set up by the American Thoracic Society and the European Respiratory Society (ATS/ERS). ${ }^{9}$ In brief, the test was performed on a marked 30-m indoor track, and subjects were told to walk as far as possible within $6 \mathrm{~min}$. The walking distance was registered at the end of the test. Subjects were allowed to take breaks during the test if needed; however, time was not interrupted. Standardized instructions and phrases of encouragement were given each minute. Oxygen supplementation was provided if required, and subjects carried their own oxygen device during the test. Walk distance is presented in meters and expressed as percent of predicted. ${ }^{10}$ Before and after the test, heart rate, and $\mathrm{S}_{\mathrm{pO}_{2}}$ were measured with a pulse oximeter (Masimo

\section{QUICK LOOK}

\section{Current knowledge}

The 6-min walk test (6MWT) is a well-established functional capacity test in lung transplant candidates. However, it is time-consuming and sensitive to variation in methodology. Growing evidence suggests the 1-min sitto-stand test (1-min STS) as an applicable and timesaving alternative to assess exercise capacity in various respiratory conditions.

\section{What this paper contributes to our knowledge}

The 1-min STS was a valid functional exercise capacity test in subjects with end-stage lung disease. The 1-min STS elicited greater dyspnea and leg fatigue compared to the 6MWT, despite fewer episodes of oxygen desaturation and comparable heart rate responses. Application of the 1-min STS in lung transplantation candidates in clinical practice may be considered as a available measure of functional exercise capacity.

Rad-5v, Masimo, Irvine, California) connected to the index finger. Ratings of perceived exertion and dyspnea were evaluated by means of a $0-10$ Borg scale. ${ }^{11}$

Knee extension strength was measured with handheld dynamometry (MicroFET2, Hoggan Industries, West Jordan, Utah) using the break technique. ${ }^{12}$ Measurements were performed by trained staff using a standardized protocol. Monthly training sessions were held to ensure standardized assessments between the assessors and to acquire highquality data. Three reproducible measurements were performed, and the highest value was used for analysis. Results were expressed as force measurements in Newtons.

The 1-min STS was performed using a standardized protocol ${ }^{3,13,14}$ on a conventional chair with no armrests and a seat height of $46 \mathrm{~cm}$. For safety reasons, the chair was positioned against a wall. The subjects were instructed to stand up and sit down as often as possible at a selfchosen speed for $1 \mathrm{~min}$, and the number of sit-to-stand repetitions was counted. Verbal encouragement was not provided during the test, but subjects were informed when $15 \mathrm{~s}$ were left until the test terminated at $60 \mathrm{~s} .{ }^{3,4,13,14}$ Subjects were allowed to stop at any time during the test. A repetition was defined as the legs being completely straight at the end of the stand-up phase, and the bottom had to have clear contact with the chair when sitting down. Subjects were told to place their hands at the hips and were not allowed to use their hands or arms to assist movement. Measurements of heart rate, $\mathrm{S}_{\mathrm{pO}_{2}}$, and Borg were performed before and after the 1-min STS using the same assessment tools as for the 6MWT. The number of 1-min STS repe- 


\section{1-Min STS IN Lung TRansplant CANDidates}

titions were expressed as percent of predicted values according to Strassmann et al. ${ }^{14}$

Allometric scaling was used to account for the effect of body weight on 6MWT and 1-min STS performance, ${ }^{15}$ as well as knee extensor strength. ${ }^{15-19}$ We used an allometric parameter of -0.33 for the 1-min STS and the 6MWT, and a parameter of 0.67 for knee extensor strength. ${ }^{15-17}$

\section{Statistical Analysis}

Pearson correlation coefficients were used to compare the results between the 1-min STS and the 6MWT exercise tests and knee extensor strength. The strength of correlation was expressed according to published recommendations. ${ }^{20}$ Non-parametric Wilcoxon signed-rank tests were used to compare pre-post exercise changes in dyspnea and leg fatigue, $\mathrm{S}_{\mathrm{pO}_{2}}$, and heart rate between the 1-min STS and the 6MWT. Oxygen desaturation was defined as a drop of $\geq 4 \%$ in $\mathrm{S}_{\mathrm{pO}_{2}}$ comparing pre- and post-test values. ${ }^{21}$ Statistical analyses were performed using SPSS Version 25 (IBM Armonk, New York).

\section{Results}

Fifty-one subjects participated in the lung transplantation evaluation. Of those, 38 had complete 1-min STS, 6MWT, and knee extensor strength data. Thirteen subjects did not perform all functional capacity tests and were excluded for the following reasons: 6MWT ( $n=6$ for severe breathlessness, $n=2$ for pain in lower extremities during walking); knee extensor strength ( $n=4$ for pain or orthopedic problems in the lower extremities); 1-min STS $(n=1$ for pain during sit-to-stand movement). The study sample consisted of subjects with interstitial lung disease (47\%), COPD (37\%), and cystic fibrosis (16\%). Baseline characteristics are presented in Table 1.

We found strong correlations between the non-normalized values of the 1-min STS and the 6MWT $(r=0.71$, $P<.001)$ and moderately strong correlations between the 1 -min STS and knee extensor strength $(\mathrm{r}=0.53, P=.001)$ and between the 6MWT and knee extensor strength ( $\mathrm{r}=0.53, P=.001$ ) (Fig. 1 ). When expressed in normalized values, correlations between the 1-min STS and the 6MWT were strong ( $\mathrm{r}=0.79, P<.001)$, whereas correlations were moderate between the 1-min STS and knee extensor strength $(\mathrm{r}=0.53, P=.001)$ and between the 6MWT and knee extensor strength $(\mathrm{r}=0.44, P=.008)$ (Fig. 1).

Significant increases in heart rate, dyspnea, and leg fatigue were observed during the 1-min STS and the 6MWT, respectively (all $P<0.001$ ). Further, $\mathrm{S}_{\mathrm{pO}_{2}}$ decreased significantly during the 1 -min STS and 6MWT $(P<0.001)$.

In direct comparison, the 1-min STS elicited significantly greater dyspnea $(P=.008)$ and leg fatigue $(P=.004)$ compared to the 6MWT. The change in $\mathrm{S}_{\mathrm{pO}_{2}}$ from pre- to post-exercise was significantly lower after the 1-min STS compared to the 6MWT $(P<.001)$. Oxygen desaturation (ie, change in $\mathrm{S}_{\mathrm{pO}_{2}} \geq 4 \%$ ) was found in $29(77 \%)$ subjects during the 1-min STS, and in $35(92 \%)$ subjects during the 6MWT. The 1-min STS induced no oxygen desaturation in 7 (18\%) subjects, in whom the 6MWT did; conversely, the 1-min STS induced oxygen desaturation in 1 (3\%) subject in whom the 6MWT did not (Table 2). No significant difference between the tests was observed in heart rate responses $(P=.241)$.

\section{Discussion}

This is the first study to evaluate the construct validity of the 1-min STS as a measure of functional exercise capacity in lung transplantation candidates. We found a strong positive correlation between the 1-min STS and the 6MWT, which suggests that the 1-min STS may be a useful and easily applicable functional capacity test in patients with end-stage lung disease. The observed correlations between the 1-min STS and 6MWT $(r=0.79)$ are within the range of those previously reported in subjects with COPD $(r=0.47-0.86), 3,6$ and higher than those reported in subjects with interstitial lung disease $(\mathrm{r}=0.50){ }^{8}$ Differences in study population characteristics (eg, age, sex, lung disease severity), sample size, as well as testing and normalization methodology may explain the differences in correlation strength between studies.

Despite the fact that our subjects had end-stage lung disease (mean $\mathrm{FEV}_{1} 31 \%$ predicted), 1-min STS performance was comparable to previous studies in subjects with COPD and interstitial lung disease with better lung function. ${ }^{3,8,22,23}$ Previous work in subjects with COPD suggests that greater age and dyspnea level, low physical activity, female sex, long-term oxygen treatment, and quadriceps muscle strength are associated with lower 1-min STS performance. ${ }^{23,24}$ Thus, the degree of airway obstruction may not necessarily affect 1-min STS performance substantially per se.

Interestingly, our subjects (of whom $50 \%$ had interstitial lung disease) experienced greater dyspnea and leg fatigue after the 1-min STS compared to the 6MWT, despite lower rates of oxygen desaturation and comparable heart rate responses. These observations are in line with those reported in subjects with interstitial lung disease. ${ }^{8}$ Greater perception of leg fatigue after the 1-min STS was also observed in subjects with COPD and was hypothesized to result from eccentric muscle contractions during the standto-sit phase. ${ }^{3}$

The 1-min STS is a responsive test ${ }^{3,4,23}$ and is able to quantify changes in functional exercise capacity after pulmonary rehabilitation, similar to the $6 \mathrm{MWT} .^{3,23}$ This makes the 1-min STS attractive for pulmonary rehabilitation set- 
Table 1. Participants' Characteristics and Functional Exercise Capacity Data

\begin{tabular}{|c|c|c|}
\hline Variables & Value & $\%$ Predicted \\
\hline Age, y & $56(43-59)$ & NA \\
\hline Sex, male/female & $23 / 15$ & NA \\
\hline Body mass index, $\mathrm{kg} / \mathrm{m}^{2}$ & $24.8(20.3-27.2)$ & NA \\
\hline \multicolumn{3}{|l|}{ Diagnosis } \\
\hline Cystic fibrosis & 6 & NA \\
\hline COPD & 14 & NA \\
\hline Interstitial lung disease & 18 & NA \\
\hline $\mathrm{FEV}_{1}, \mathrm{~L}$ & $0.87(0.63-1.64)$ & $31(21-43)$ \\
\hline FVC, L & $1.95(1.51-2.58)$ & $46(37-60)$ \\
\hline \multicolumn{3}{|l|}{ Exercise capacity } \\
\hline \multicolumn{3}{|l|}{ 6-min walk test } \\
\hline Distance, $\mathrm{m}$ & $385(264-454)$ & $66(47-83)$ \\
\hline Distance, $\mathrm{m} / \mathrm{kg}$ body mass ${ }^{-0.33 *}$ & $3.93(2.67-4.66)$ & NA \\
\hline $\mathrm{S}_{\mathrm{pO}_{2}}$, pre-test, $\%$ & $95(93-96)$ & NA \\
\hline $\mathrm{S}_{\mathrm{pO}_{2}}$, post-test, $\%$ & $73(75-89)$ & NA \\
\hline Heart rate, pre-test, beats/min & $89(76-101)$ & NA \\
\hline Heart rate, post-test, beats/min & $119(107-129)$ & NA \\
\hline Dyspnea pre-post change, $0-10$ scale $\dagger$ & $5(3-7)$ & NA \\
\hline Leg fatigue pre-post change, $0-10$ scale $\dagger$ & $2(1-4)$ & NA \\
\hline \multicolumn{3}{|l|}{ 1-min sit-to-stand test } \\
\hline Repetitions, no. & $21(14-27)$ & $51(34-65)$ \\
\hline Repetitions, no./kg body mass ${ }^{-0.33 *}$ & $0.20(0.13-0.29)$ & NA \\
\hline $\mathrm{S}_{\mathrm{pO}_{2}}$, pre-test, $\%$ & $95(93-96)$ & NA \\
\hline $\mathrm{S}_{\mathrm{pO}_{2}}$, post-test, $\%$ & $88(81-92)$ & NA \\
\hline Heart rate, pre-test, beats/min & $90(80-100)$ & NA \\
\hline Heart rate, post-test, beats/min & $112(102-129)$ & NA \\
\hline Dyspnea pre-post change, $0-10$ scale $\dagger$ & $6(5-8)$ & NA \\
\hline Leg fatigue pre-post change, $0-10$ scale $\dagger$ & $4(2-5)$ & NA \\
\hline \multicolumn{3}{|l|}{ Dynamometry } \\
\hline Knee extension strength, Newtons & $279(206-361)$ & NA \\
\hline Knee extension strength* (Newtons/kg body mass ${ }^{0.67}$ ) & $7.39(6.22-9.36)$ & NA \\
\hline \multicolumn{3}{|l|}{$\begin{array}{l}\text { Data are median (interquartile range) or } n . N=38 \text { subjects. } \\
\text { * Data are normalized for body mass. } \\
\dagger \text { Dyspnea and leg fatigue were assessed with the } 0-10 \text { Borg scale. } \\
\mathrm{NA}=\text { not applicable }\end{array}$} \\
\hline
\end{tabular}

tings. Although these tests measure different aspects of functional exercise capacity, both rely heavily on quadriceps muscle strength. ${ }^{25,26}$ The major advantage of the 1-min STS compared to the 6MWT is that the test requires less time and space and has a better feasibility for testing patients in need of isolation treatment due to the risk of cross-infections, such in the case of cystic fibrosis. Accordingly, the 1-min STS may be a useful and quickly available tool for general practitioners referring subjects for lung transplantation evaluation. In those settings, performance of a 6MWT according to the ATS/ERS technical standard guidelines ${ }^{9}$ is frequently not feasible due to limited space availabilities.

It is important to note that, at this stage, the 1-min STS cannot replace the established use of the 6MWT in lung transplantation candidates, which is currently part of the Lung Allocation Score in the United States. ${ }^{27}$ However, it may offer an attractive and easily applicable alternative when time and space are limited in clinical settings. Our findings provide an interesting basis for the application of the 1-min STS in rehabilitation settings. The test does not appear to provoke increased risks compared to the 6MWT because it involves similar hemodynamic stress but a shorter testing time and less oxygen desaturation than the 6MWT. Although the 1-min STS is an easily applicable test to screen for oxygen desaturation, the 6MWT likely provides more accurate information on changes in pre-post exercise $\mathrm{S}_{\mathrm{pO}_{2}}$. It is possible that we underestimated the change in $\mathrm{S}_{\mathrm{pO}_{2}}$ during the 6MWT because we did not continuously measure $\mathrm{S}_{\mathrm{pO}_{2}}$ during the 6MWT, as previously recommended. ${ }^{9}$ Previous research has shown that the lowest $\mathrm{S}_{\mathrm{pO}_{2}}$ does not always occur at the end of exercise. ${ }^{9}$ In contrast, continuous $\mathrm{S}_{\mathrm{pO}_{2}}$ measurements during the 1-min STS are hampered by movement artifacts, ${ }^{13}$ and our group has re- 

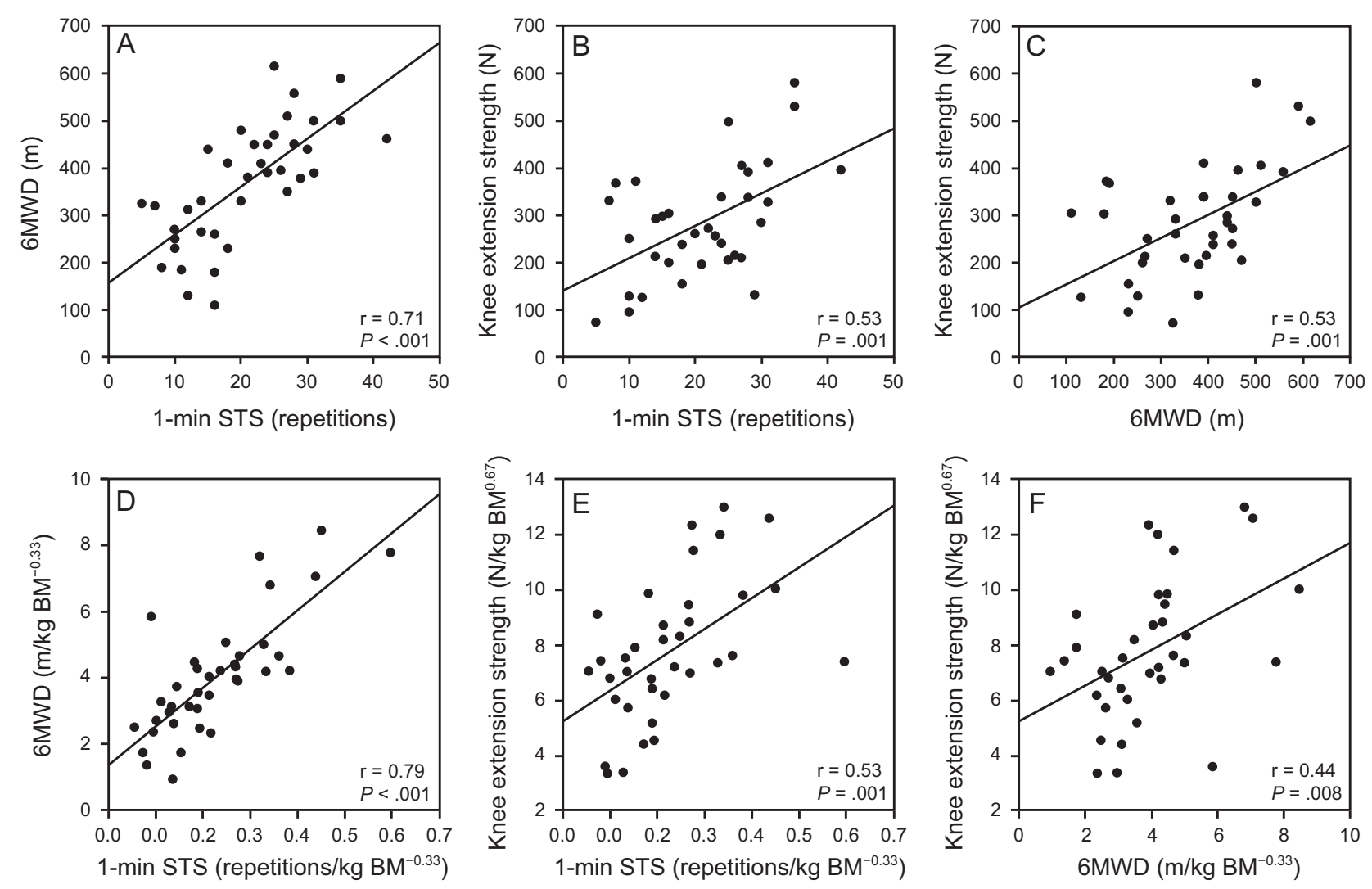

Fig. 1. Comparisons between (A) non-normalized 6MWD and 1-min STS repetitions; (B) non-normalized knee extension strength and 1-min STS repetitions; (C) non-normalized knee extension strength and 6MWD; (D) normalized 6MWD and 1-min STS repetitions; (E) normalized knee extension strength and 1-min STS repetitions; and (F) normalized knee extension strength and 6MWD. 6MWD =6-min walk distance; 1-min STS = 1-min sit-to-stand test; $\mathrm{N}=$ Newton; $\mathrm{kg} \mathrm{BM}=$ body mass in $\mathrm{kg}$.

Table 2. Oxygen Desaturation During the 6MWT and the 1-min STS

\begin{tabular}{lcr}
\hline \hline & \multicolumn{2}{c}{$\Delta \mathrm{S}_{\mathrm{pO}_{2}} \geq 4 \%$ during 6MWT } \\
\cline { 2 - 3 } & \multicolumn{1}{c}{ No } & \multicolumn{1}{c}{ Yes } \\
\hline$\Delta \mathrm{S}_{\mathrm{pO}_{2}} \geq 4 \%$ during 1-min STS & & \\
No & $2(5)$ & $7(18)$ \\
Yes & $1(3)$ & $28(74)$ \\
& & \\
Data are presented as $n$ (\%). & & \\
6MWT = 6-min walk test & & \\
1-min STS $=$ 1-min sit-to-stand test & & \\
\hline
\end{tabular}

cently shown that the lowest $\mathrm{S}_{\mathrm{pO}_{2}}$ often occurs during the first minute after the sit-to-stand exercise. ${ }^{3,13}$ To our knowledge, no previous study has investigated the prognostic value of oxygen desaturation during the 1-min STS, as previously reported for the $6 \mathrm{MWT} .^{28}$

In our study, the 1-min STS and the 6MWT showed similar correlations with knee extensor strength. Previous work in subjects with cystic fibrosis ${ }^{29}$ and $\mathrm{COPD}^{7}$ with a broad range of disease severity found no correlations between the 1-min STS and quadriceps muscle force mea- sured with the same handheld dynamometry device as that used in our study when using absolute values, ${ }^{7}$ and moderately strong correlations when expressed as a product of body weight. ${ }^{29}$ However, another study using the same device and measurement technique reported correlations similar to ours in subjects with moderate COPD. ${ }^{23}$ Further studies in individuals with $\mathrm{COPD}^{3,30}$ and cystic fibrosis ${ }^{31}$ using different devices have reported correlations similar to ours. We can only speculate on the underlying reasons for the differences between the studies. Generalized interpretation of measurements of functional exercise capacity should ideally be made with adjustments to body mass, independent of the population under study. ${ }^{9,15,18,19,32}$ If adjustments for body mass are taken into account, correlations between the 1-min STS and knee extensor strength appear to attenuate. ${ }^{29}$ Additionally, limb length may be considered through the assessment of torque instead of force. In our study, the moment arm of the knee extensor was not assessed, and normalization with respect to limb length was not possible. Furthermore, due to the wellknown learning effect for the 6MWT, 2 tests are recommended and the test with the longest walking distance should be used as a measure of functional capacity. ${ }^{9}$ At our 


\section{1-Min STS in Lung Transplant CANDidates}

institution, lung transplantation candidates usually perform only one walking test as part of the lung transplantation listing assessments. However, because our subjects were familiar with the 6MWT in the clinical setting, learning effects can be ruled out.

In summary, functional exercise capacity seems to rely consistently on lower limb strength in end-stage pulmonary disease and could accordingly provide a simple means to measure and monitor peripheral muscle (dys)function as part of pre- and post-lung transplantation rehabilitation programs.

This is a small-sized exploratory study. Our data need to be confirmed in larger patient populations undergoing lung transplantation evaluation. Further research should investigate the prognostic value of the 1-min STS in lung transplantation candidates along with a head-to-head comparison to the 6MWT. To date, comparisons of the 2 tests after lung transplantation are not available; however, such comparisons may provide further insights into the diagnostic value of functional exercise capacity assessments in lung transplantation candidates.

\section{Conclusions}

The 1-min STS is a valid test in patients with end-stage lung disease. Due to its ease of application, medical practitioners may consider using the 1-min STS in situations when the 6MWT cannot be performed (eg, time limitations, or limited space availability). Because the 1-min STS causes less oxygen desaturation than the 6MWT and comparable heart rate responses compared to the 6MWT, it may be considered a safe test in patients with end-stage lung disease.

\section{REFERENCES}

1. Weill D, Benden C, Corris PA, Dark JH, Davis RD, Keshavjee S, et al. A consensus document for the selection of lung transplant candidates: 2014-an update from the Pulmonary Transplantation Council of the International Society for Heart and Lung Transplantation. J Heart Lung Transplant 2015;34(1):1-15.

2. Singh SJ, Puhan MA, Andrianopoulos V, Hernandes NA, Mitchell $\mathrm{KE}$, Hill CJ, et al. An official systematic review of the European Respiratory Society/American Thoracic Society: measurement properties of field walking tests in chronic respiratory disease. Eur Respir J 2014;44(6):1447-1478.

3. Crook S, Busching G, Schultz K, Lehbert N, Jelusic D, Keusch S, et al. A multicentre validation of the 1-min sit-to-stand test in patients with COPD. Eur Respir J 2017;49(3).

4. Radtke T, Puhan MA, Hebestreit H, Kriemler S. The 1-min sit-tostand test: a simple functional capacity test in cystic fibrosis? J Cyst Fibros 2016;15(2):223-226.

5. Crook S, Frei A, Ter Riet G, Puhan MA. Prediction of long-term clinical outcomes using simple functional exercise performance tests in patients with COPD: a 5-year prospective cohort study. Respir Res 2017;18(1):112.

6. Vaidya T, Chambellan A, de Bisschop C. Sit-to-stand tests for COPD: a literature review. Respir Med 2017;128:70-77.
7. Reychler G, Boucard E, Peran L, Pichon R, Le Ber-Moy C, Ouksel $\mathrm{H}$, et al. One minute sit-to-stand test is an alternative to 6MWT to measure functional exercise performance in COPD patients. Clin Respir J 2018;12(3):1247-1256.

8. Briand J, Behal H, Chenivesse C, Wemeau-Stervinou L, Wallaert B. The 1-minute sit-to-stand test to detect exercise-induced oxygen desaturation in patients with interstitial lung disease. Ther Adv Respir Dis 2018;12:1753466618793028.

9. Holland AE, Spruit MA, Troosters T, Puhan MA, Pepin V, Saey D, et al. An official European Respiratory Society/American Thoracic Society technical standard: field walking tests in chronic respiratory disease. Eur Respir J 2014;44(6):1428-1446.

10. Chetta A, Zanini A, Pisi G, Aiello M, Tzani P, Neri M, et al. Reference values for the 6-min walk test in healthy subjects 20-50 years old. Respir Med 2006;100(9):1573-1578.

11. Gift AG, Narsavage G. Validity of the numeric rating scale as a measure of dyspnea 1998;7(3):200-204.

12. Douma RK, Soer R, Krijnen WP, Reneman M, van der Schans CP. Reference values for isometric muscle force among workers for the Netherlands: a comparison of reference values. BMC Sports Sci Med Rehabil 2014;6(1):10.

13. Radtke T, Hebestreit H, Puhan MA, Kriemler S. The 1-min sit-tostand test in cystic fibrosis - Insights into cardiorespiratory responses. J Cyst Fibros 2017;16(6):744-751.

14. Strassmann A, Steurer-Stey C, Lana KD, Zoller M, Turk AJ, Suter $\mathrm{P}$, et al. Population-based reference values for the 1-min sit-to-stand test. Int J Public Health 2013;58(6):949-953.

15. Jaric S, Mirkov D, Markovic G. Normalizing physical performance tests for body size: a proposal for standardization. J Strength Cond Res 2005;19(2):467-474.

16. Jaric S. Muscle strength testing. Sports Medicine 2002;32(10):615-631.

17. Jaric S, Radosavljevic-Jaric S, Johansson H. Muscle force and muscle torque in humans require different methods when adjusting for differences in body size. Eur J Appl Physiol 2002;87(3):304-307.

18. Beauchamp MK, Sibley KM, Lakhani B, Romano J, Mathur S, Goldstein RS, et al. Impairments in systems underlying control of balance in COPD. Chest 2012;141(6):1496-1503.

19. Davies MJ, Dalsky GP. Normalizing strength for body size differences in older adults. Med Sci Sports Exerc 1997;29(5):713-717.

20. Evans JD. Straightforward statistics for the behavioral sciences. ýPacific Grove, CA: Brooks/Cole Publishing; 1996.

21. Hadeli KO, Siegel EM, Sherrill DL, Beck KC, Enright PL. Predictors of oxygen desaturation during submaximal exercise in 8,000 patients. Chest 2001;120(1):88-92.

22. van Gestel AJ, Clarenbach CF, Stowhas AC, Rossi VA, Sievi NA, Camen G, et al. Predicting daily physical activity in patients with chronic obstructive pulmonary disease. PLoS One 2012;7(11):e48081.

23. Vaidya T, de Bisschop C, Beaumont M, Ouksel H, Jean V, Dessables $\mathrm{F}$, et al. Is the 1-minute sit-to-stand test a good tool for the evaluation of the impact of pulmonary rehabilitation? Determination of the minimal important difference in COPD. Int J Chron Obstruct Pulmon Dis 2016;11:2609-2616.

24. Crook S, Frei A, Yu T, Ter Riet G, Puhan M. Baseline determinants of 1-minute sit-to-stand test performance in COPD patients. Eur Respir J 2015;46(suppl 59):PA3713.

25. Butcher SJ, Pikaluk BJ, Chura RL, Walkner MJ, Farthing JP, Marciniuk DD. Associations between isokinetic muscle strength, highlevel functional performance, and physiological parameters in patients with chronic obstructive pulmonary disease. Int J Chron Obstruct Pulmon Dis 2012;7:537-542.

26. Singer J, Yelin EH, Katz PP, Sanchez G, Iribarren C, Eisner MD, et al. Respiratory and skeletal muscle strength in chronic obstructive pulmonary disease: impact on exercise capacity and lower extremity function. J Cardiopulm Rehabil Prev 2011;31(2):111-119. 


\section{1-Min STS IN Lung TRansplant CANDidates}

27. Castleberry A, Mulvihill MS, Yerokun BA, Gulack BC, Englum B, Snyder L, et al. The utility of 6-minute walk distance in predicting waitlist mortality for lung transplant candidates. J Heart Lung Transplant 2017;36(7):780-786.

28. Casanova C, Cote C, Marin JM, Pinto-Plata V, de Torres JP, Aguirre-Jaime A, et al. Distance and oxygen desaturation during the 6-min walk test as predictors of long-term mortality in patients with COPD. Chest 2008;134(4):746-752.

29. Gruet M, Peyre-Tartaruga LA, Mely L, Vallier JM. The 1-minute sit-to-stand test in adults with cystic fibrosis: correlations with cardiopulmonary exercise test, 6-minute walk test, and quadriceps strength. Respir Care 2016;61(12):1620-1628.
30. Rausch-Osthoff AK, Kohler M, Sievi NA, Clarenbach CF, van Gestel AJ. Association between peripheral muscle strength, exercise performance, and physical activity in daily life in patients with chronic obstructive pulmonary disease. Multidiscip Respir Med 2014;9(1):37.

31. Sheppard E, Chang K, Cotton J, Gashgarian S, Slack D, Wu K, et al. Functional tests of leg muscle strength and power in adults with cystic fibrosis. Respir Care 2019;64(1):40-47.

32. Maltais F, Decramer M, Casaburi R, Barreiro E, Burelle Y, Debigare $\mathrm{R}$, et al. An official American Thoracic Society/European Respiratory Society statement: update on limb muscle dysfunction in chronic obstructive pulmonary disease. Am J Respir Crit Care Med 2014;189(9):e15-e62.

This article is approved for Continuing Respiratory Care Education credit. For information and to obtain your CRCE

(free to AARC members) visit

www.rcjournal.com 